\title{
Kidney growth and hypertrophy: the role of mTOR and vesicle trafficking
}

\author{
Qais Al-Awqati \\ Departments of Medicine and Physiology and Cellular Biophysics, College of Physicians and Surgeons of Columbia University, New York, New York, USA.
}

\begin{abstract}
The kidney, like other organs, grows in constant proportion to the rest of the body. When one kidney is removed, the remaining one hypertrophies. In a comprehensive series of studies, Chen et al. show that growth during maturation is mediated by the mTORC1 signaling pathway, which is induced by ECF-like peptides, and requires PI3K, PDK, AKT, mTORC2, and activation of mTORC1 through the combined effects of TSC and RHEB as part of a multiprotein complex localized on lysosomes. However, compensatory growth is mediated by amino acids, which act on mTORC1 independently of the previous pathway, and requires a class III PI3K (VPS34) that is known to be involved in vesicle trafficking to the lysosomes.
\end{abstract}

The mechanism that guarantees organ growth in proportion to the body has attracted the attention of a wide array of scientists since ancient times (1). For many organs, growth is mediated by individual, organ-specific mechanisms, which are often context dependent. The kidney seems to be a special case, because it is not an organ in the same way that the heart is an organ. Removal of half a kidney reduces its function by half, while removal of half of the heart eliminates all function. It is the nephron that is the organ, and in vertebrates, all these units are packaged in one place - the kidney. Removal of one kidney causes a compensatory hypertrophy of the remaining organ, but unfortunately without increasing the number of nephrons, as human nephrogenesis ceases at birth (2).

During maturation, cell proliferation participates in the increase in size and length of nephrons, but cell hypertrophy is a major component of the increase in renal size both during maturation and especially during compensatory hypertrophy. While each nephron hypertrophies after birth, renal size continues to maintain a remarkably constant relationship to that of the

Related Article: p. 2429 body as both grow toward their adult size. Nephron function also increases; at birth, human glomerular filtration rate (GFR) is very low, but at the end of infancy, the $\mathrm{GFR} / \mathrm{m}^{2}$ surface area remains constant (1) as children grow to adulthood, meaning that in childhood, GFR increases proportionately to body size. Elegant microdissection studies have shown that during both maturational growth and in compensatory hypertrophy, the largest growth occurs in the proximal tubules (3). Could a single mechanism mediate maturational growth and compensatory hypertrophy? In this issue, Chen et al. (4) suggest that the two are related and provide compelling evidence for the role of the anabolic signaling pathway mediated by mTORC1 in both types of growth.

\section{Receptor tyrosine kinases mediate renal growth during maturation}

Recently, the mTOR kinase has emerged as the central enzyme that regulates cell growth following binding of growth factors to their receptor tyrosine kinases (RTKs) (5). Binding of a ligand to an RTK such as EGFR activates PI3K, which

Conflict of interest: The author has declared that no conflict of interest exists.

Reference information: J Clin Invest. 2015;125(6):2267-2270. doi:10.1172/JCI81508.

phosphorylates PIP2 to PIP3. PIP3 activates PDK1 and mTORC2, which starts a phosphorylation cascade that activates AKT, which then phosphorylates the tuberous sclerosis (TSC) proteins, which inhibit RHEB, a small GTPase that is a tonic inhibitor of mTORC1. That pathway (RTK/PDK1/mTORC2/AKT/TSC/ RHEB mTORC1 [herein referred to as RTK/mTORC1 for simplicity]) is the major mechanism by which growth factors such as insulin, EGF, and others promote anabolic activity through activation of mTORC1, which phosphorylates S6K1 and thereby promotes protein synthesis. PTEN is a phosphatase that normally converts PIP3 back to PIP2, thereby nullifying the effect of PI3K. Chen et al. (4) found that deletion of Pten from the proximal tubule (thereby removing the brake on this pathway) results in proximal tubular hypertrophy during maturation. When both Egfr and Pten were deleted, the kidney was smaller in size, and activation of the components of the RTK/mTORC1 pathway was reduced. However, hypertrophy was not eliminated but was attenuated, and compensatory hypertrophy was unaffected, suggesting that another pathway might activate mTORC1 (see Figure 1).

\section{Increased amino acid delivery causes compensatory hypertrophy}

In the $19^{\text {th }}$ century, the cause of compensatory hypertrophy was proposed to be due to an increased work load of the remaining kidney. Moreover, at this time, renal work was assumed to be the excretion of nitrogenous wastes, a notion that was supported by the finding that a high-protein diet caused kidney hypertrophy (1). More recently, the immediate increase in renal blood flow and GFR that is always seen after uninephrectomy or high-protein diets has been implicated as the cause of hypertrophy. However, the relations between increased GFR, blood flow, and 


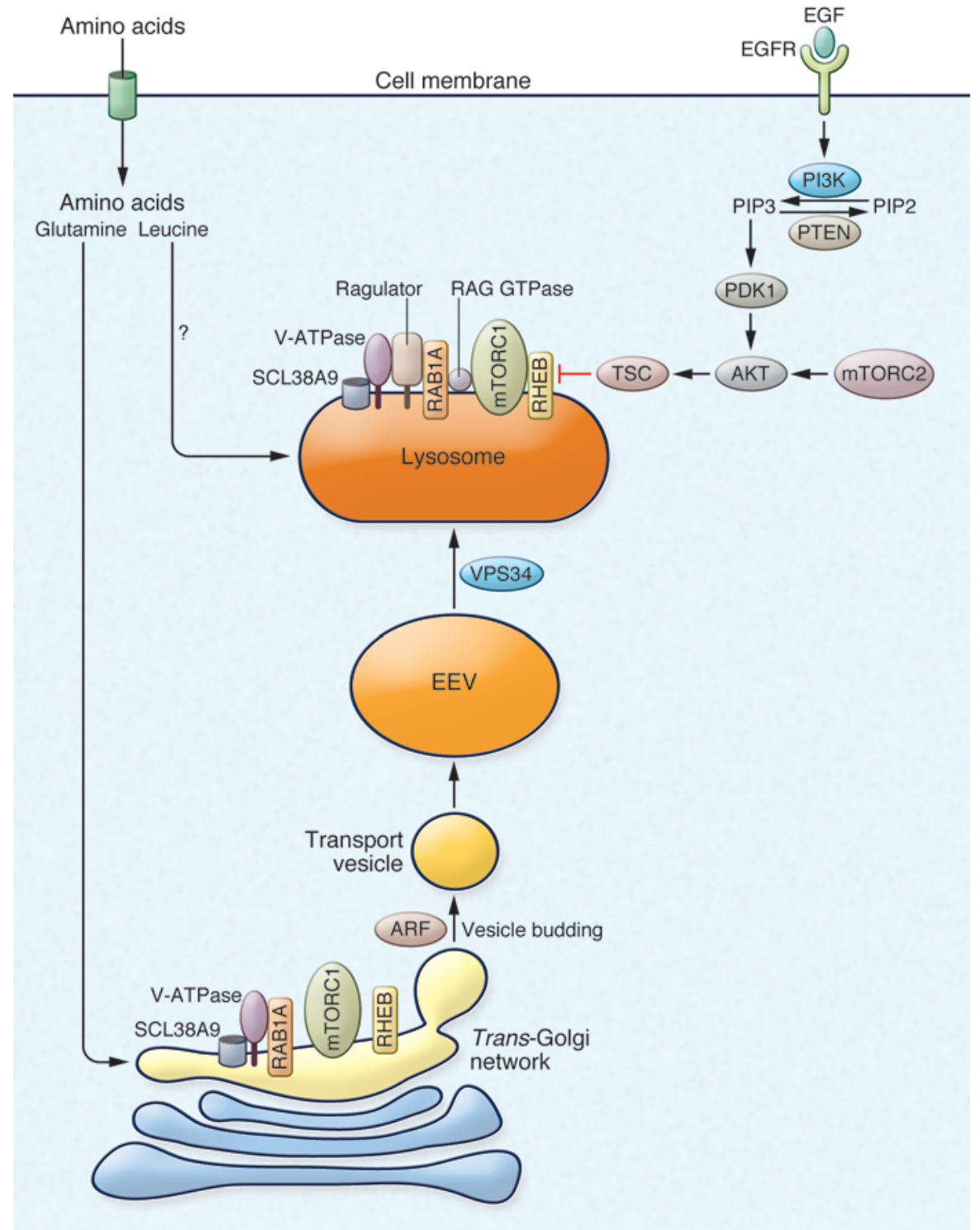

hypertrophy were somewhat confusing $(6,7)$. Chen et al. (4) finally clarify these issues and demonstrate that following uninephrectomy, the immediate increase in renal blood flow augments proximal tubular amino acid content, presumably due to absorption of filtered amino acids. Indeed, infusion of amino acids, independent of uninephrectomy, stimulated anabolic activity. It is this increased delivery that directly activates mTORC1 by a mechanism that differs from that mediated by RTK activation and is responsible for maturational hypertrophy.

It is known from in vitro studies that mTORC1 can be stimulated by amino acids to induce growth in a manner that is independent of the response to RTK signaling (5). mTORC1 becomes activated only when present in a very large multicomponent complex, which senses the presence of specific amino acids (glutamine, leucine, and arginine) in the lumen of lysosomes. This complex begins to form when the RAG GTPases are activated and bind to their regulator (Ragulator), the vacuolar proton pump (V-ATPase), and the GATOR complex. When this complex is formed, the RAG GTPases recruit mTORC1 to the lysosomal membrane. Recently, the amino acid transporter SLC38A9 was identified as a necessary component of this complex that confers amino acid sensitivity (8, 9). Acidification of the lysosomal lumen by V-ATPase is also necessary, although the role low $\mathrm{pH}$ plays is obscure. Once the mTORC1-containing complex is recruited to the lysosome, it encounters RHEB, whose inhibition activates it.
Figure 1. Maturation and compensatory kidney growth activate mTORC1 through different mechanisms. Growth factor (EGF) binding to its respective RTK (EGFR) activates PI3K, resulting in conversion of PIP2 to PIP3. In turn, PIP3 initiates a PDK1-dependent cascade that activates AKT, which in turn phosphorylates TSC, resulting in inhibition of the tonic mTORC1 inhibitor RHEB. mTORC1 is recruited into a large protein complex on the lysosome surface composed of RAG GTPase, Ragulator, V-ATPase, and the amino acid transporter SLC38A9, allowing mTORC1 to be activated via inhibition of RHEB. Constitutive activation of this pathway via deletion of PTEN, which converts PIP3 to PIP2, results in proximal tubular hypertrophy during maturation. Uninephrectomy causes an immediate increase in renal blood flow, which enhances renal amino acid content. These amino acids activate mTORC1 by a process requiring class III PI3K (VPS34), which is involved in endocytosis and vesicle recycling from the Golgi to the lysosome. Several recent studies have implicated intracellular membrane trafficking in the process by which some amino acids activate the mTORC1 pathway. mTORC1 is present in the ER and TGN, and the complex appears to traffic to the lysosome via vesicles, as its activation is blocked by loss of RAB1A, known to regulate ER-to-Golgi transport, or ARF, known to mediate budding from the Golgi. Transport to the lysosome likely occurs via a VPS34-mediated pathway. The TGN is slightly acidic $(\sim \mathrm{pH}=6.4$, yellow), and as vesicles move to the lysosome ( pH $=4.5-5$, dark orange), they become increasingly acidic. It is not clear whether mTORC1 can be activated by amino acids when in the Golgi without prior transport to the lysosome.

\section{Golgi-to-lysosome vesicle trafficking and mTORC1 activation}

One of the more remarkable findings of the paper by Chen et al. (4) is their demonstration that the mechanism by which amino acids stimulate mTORC1 requires VPS34 (class III PI3K), an enzyme that is involved in endocytosis and vesicle recycling from the Golgi to the surface (10). What could vesicle recycling and the Golgi have to do with activation of mTORC1 on the surface of the lysosome? A slew of recent studies $(11,12)$ have implicated intracellular membrane trafficking in the process by which some amino acids activate the mTORC1 pathway (Figure 1). For instance, glutamine activation of mTORC1 does not require RAG GTPases, while leucine does (11). Others found that in RAG-deficient 
cells, mTORC1 is localized to the Golgi, and this localization is dependent on ARF, an ADP-ribosylating factor critical for vesicle trafficking (11). Further, while addition of glutamine to RAG-deficient cells caused translocation of mTORC1 to the lysosomes, it took much longer to localize there (11). Previous studies have also shown that mTORC1 is present in the ER as well as in the trans-Golgi network (TGN) (13).

How do proteins get targeted to lysosomes? Lysosomal hydrolases are synthesized in the ER and glycosylated in the Golgi, where they acquire a mannose-6phosphate ligand (14). When lysosomal hydrolases reach the TGN, they bind to mannose-6-phosphate receptors (M6PRs) and bud off into clathrin-coated vesicles, a process mediated by several adaptor proteins and facilitated by ARF1. The vesicles then fuse with early endosomal vesicles (EEVs) and eventually traffic and fuse with the lysosomes, delivering the lysosomal hydrolases to their final destination. When cells lacking RAG GTPase were treated with brefeldin, amino acid-induced activation of mTORC1 was abolished, but brefeldin did not prevent mTORC1 activation in RAG GTPase-sufficient cells (11). Brefeldin inhibits activation of ARF1, causing blockade of anterograde transport from the ER to the Golgi. Thus, activation of mTORC1 requires normal ER-toGolgi transport. Further, it was found that knockout of Rab1a, a small GTPase involved in endocytosis, also results in blockade of mTORC1 activation by amino acids (12). While RAB1A was previously only known for its control of ER-to-Golgi transport, it was found to induce association of mTORC1 with RHEB and RAPTOR, independently of RAG GTPases. Further, knockdown of RAB1A interfered with mTORC1 localization in the Golgi but not in the lysosomes. Moreover, loss of RAB1A did not affect mTORC1 interaction with RAG GTPases in the lysosome (13).

Two possibilities emerge from these new studies. One possibility is that mTORC1 can only be active when it is located on lysosomal membranes but can be delivered to the lysosome from other intracellular membranes such as the TGN in an inactive form. The other possibility is that mTORC1 can be activated, by amino acids for example, when it is on the Golgi or other membrane compartments. It is difficult to conclude one way or another on the basis of currently available data, as mTORC1 is present in the secretory pathway in association with many of its binding/activating proteins (13). To distinguish between these possibilities, it will be necessary to obtain careful kinetic analysis of the process of mTORC1 activation. To my knowledge, only Jewell et al. (11) have studied the kinetics of mTORC1 activation. This group has shown that in RAG-sufficient cells, glutamine causes mTORC1 to localize to lysosomes within 50 minutes of addition, while in RAGdeficient cells, mTORC1 is not in the lysosome at 50 minutes, but is present at 150 minutes after the addition of glutamine. However, Jewell et al. did not identify the compartment where mTORC1 was localized before it arrived in the lysosome, nor did they determine whether mTORC1 was active in that locale. It will be important to show that glutamine causes localization of the complex to the Golgi and to determine whether it is active in that locale. Interestingly, mTORC1 in one study was found to colocalize with golgin 97 (13), a protein known to associate with M6PR (15).

Thus, it appears that the mTORC1 activation complex gets loaded onto the TGN following addition of glutamine, perhaps in association with SLC38A9, which transports glutamine (but not leucine) (Figure 1). Further, this region of the Golgi is acidified by the V-ATPase; hence, it will likely bind to the mTORC1 complex as well as with RAPTOR and RHEB, the latter of which is already known to exist in the Golgi (16). This complex may then be shuttled by vesicles to the lysosomes through the welldescribed common highway taken by the M6PR to the lysosomes. The major question is whether mTORC1 is active while resident on Golgi membranes or needs to traffic to the lysosome to become active. Here, Chen et al., in a critical study, found that deletion of Vps34 blocked amino acidmediated activation of mTORC1. What might be the specific function of this class III PI3K in mTORC1 trafficking? Recently, a highly specific inhibitor of VPS34 was found to block trafficking of vesicles from the TGN to the lysosome (17). Thus, it can be speculated that mTORC1 is not active when it is in the Golgi but can only be activated when it reaches the lysosome. If this is the case, it will be important to deter- mine what component of the complex is supplied by the lysosomes and is so necessary for activation.

The kidney has provided a nice in vivo model for the separation of RTK from amino acid pathways. We can finally rationalize the ancient findings of high-protein diet feeding with modern molecular cell biology; yet both processes are mediated by mTORC1.

Address correspondence to: Qais Al-Awqati, Departments of Medicine and Physiology and Cellular Biophysics, College of Physicians and Surgeons of Columbia University, $630 \mathrm{~W} 168^{\text {th }}$ St., New York, New York 10032, USA. E-mail: qa1@cumc.columbia.edu.

1. Smith HW. Renal function in infancy and childhood. In: Smith HW. The Kidney: Structure and Function in Health and Disease. New York, New York, USA: Oxford University Press; 1951:492-519.

2. Oliver J. Nephrons and Kidneys. New York, New York, USA: Hoeber Medical Division of Harper and Row; 1968.

3. Oliver J. New directions in renal morphology: a method, its results and its future. Harvey Lect. 1944-1945;40:102-155.

4. Chen J-K, et al. Phosphatidylinositol 3-kinase signaling determines kidney size. JClin Invest. 2015;125(6):2429-2444

5. Laplante M, Sabatini DM. mTOR signaling in growth control and disease. Cell. 2012;149(2):274-293.

6. Katz AI, Epstein FH. Relation of glomerular filtration rate and sodium reabsorption to kidney size in compensatory renal hypertrophy. Yale J Biol Med.1967;40(3):222-230.

7. Katz AI, Toback FG, Lindheimer MD. Independence of onset of compensatory kidney growth from changes in renal function. Am J Physiol. 1976;230(4):1067-1071.

8. Wang S, et al. Lysosomal amino acid transporter SLC38A9 signals arginine sufficiency to mTORC1. Science. 2015;347(6218):188-194.

9. Rebsamen M, et al. SLC38A9 is a component of the lysosomal amino acid sensing machinery that controls mTORC1. Nature. 2015;519(7544):477-481.

10. Backer JM. The regulation and function of Class III PI3Ks: novel roles for Vps34. Biochem J. 2008;410(1):1-17.

11. Jewell JL, et al. Differential regulation of mTORC1 by leucine and glutamine. Science. 2015;347(6218):194-198.

12. Thomas JD, et al. Rab1A is an mTORC1 activator and a colorectal oncogene. Cancer Cell. 2014; 26(5):754-769.

13. Drenan RM, Liu X, Bertram PG, Zheng XF. FKBP12-rapamycin-associated protein or mammalian target of rapamycin (FRAP/ $\mathrm{mTOR}$ ) localization in the endoplasmic reticulum and the Golgi apparatus. J Biol Chem. 
2004;279(1):772-778.

14. Ghosh P, Dahms NM, Kornfeld S. Mannose 6-phosphate receptors: new twists in the tale. Nat Rev Mol Cell Biol. 2003;4(3):202-212.

15. Wong M, Munro S. Membrane trafficking.
The specificity of vesicle traffic to the Golgi is encoded in the golgin coiled-coil proteins. Science. 2014;346(6209):1256898.

16. Yadav RB, et al. mTOR direct interactions with Rheb-GTPase and raptor: sub-cellular localiza- tion using fluorescence lifetime imaging. $B M C$ Cell Biol. 2013;14:3.

17. Ronan B, et al. A highly potent and selective Vps34 inhibitor alters vesicle trafficking and autophagy. Nat Chem Biol. 2014;10(12):1013-1019. 